\title{
Characterization of AMPylation on Threonine, Serine, and Tyrosine Using Mass Spectrometry
}

\author{
Yan Li, ${ }^{1}$ Rowaida Al-Eryani, ${ }^{1}$ Melanie L. Yarbrough, ${ }^{2}$ Kim Orth, ${ }^{2}$ Haydn L. Ball ${ }^{1}$ \\ ${ }^{1}$ Protein Chemistry Technology Center, Department of Internal Medicine, University of Texas, Southwestern Medical Center, \\ Dallas, TX USA \\ ${ }^{2}$ Department of Molecular Biology, University of Texas, Southwestern Medical Center, Dallas, TX USA
}

\begin{abstract}
Recent studies have suggested that adenosine $5^{\prime}$-monophosphate (AMP) post-translational modification of proteins could represent a novel molecular signaling pathway. Mass spectrometric fragmentation characteristics of this modification have not previously been described and studied systematically. In this work, we therefore examined the fragmentation pattern of chemically synthesized peptides containing AMPylated Thr, Ser, and Tyr. The formation of characteristic ions and the influence of collision energy (CE) on the detection of characteristic ions and their relative peak intensity are reported. When peptide with AMPylated Ser/Thr underwent collision induced dissociation (CID), peaks at $\mathrm{m} / \mathrm{z} 348.1,136.1$, and 250.1, fragments with AMP group attached, and fragments consistent with neutral loss of $347 \mathrm{Da}$ were major characteristic ions; fragments consistent with neutral loss of $135 \mathrm{Da}$ or $249 \mathrm{Da}$ were weaker and not always detectable. The observations for Tyr AMPylation followed the same general patterns as those for Ser/Thr modification, with the exception that the ions detected for Tyr AMPylation did not include either the peak at $\mathrm{m} / \mathrm{z} 348.1$, or fragments with a mass shift of $-347 \mathrm{Da}$. The results described in this paper highlight a series of diagnostic ions, which can be used not only to confidently identify the AMPylation site based on MS and MS/MS data, but also to selectively scan AMPylated peptides in complex protein mixtures.
\end{abstract}

Key words: Post-translational modification, AMPylation, CID fragmentation, Adenylylation, Adenylation

\section{Introduction}

Secific enzymes have been shown to covalently bind adenosine 5'-monophosphate (AMP) and form enzymeAMP intermediates, a process known as adenylylation [1]. Adenylylation is an essential step in the enzymatic mechanism for DNA/RNA ligation [2]. Another term, adenylation, has also been used, especially in the field of peptide biosynthesis, and refers to the process whereby peptide synthetases activate a specific amino acid to an aminoacylO-AMP form [3, 4]. Recently, Yarbrough et al. showed that VopS, a Vibrio parahaemolyticus type III effector, can modify Rho, Rac, and Cdc42 with AMP, a process referred to as AMPylation, and inhibit their interaction with down-

Correspondence to: Haydn L. Ball; e-mail: haydn.ball@utsouthwestern.edu stream effectors [5]. They demonstrated that AMPylation could represent an important post-translational modification (PTM) involved in molecular signaling [5, 6]. Although it is known that enzymes form AMP-enzyme intermediates to drive chemical ligation reactions [7], AMP had not previously been shown to be used as a stable PTM in protein signaling. Others have reported that IbpA and DrrA can add an AMP to a Tyr residue in Rho GTPases $[8,9]$. Their study also suggested that addition of AMP may be a PTM that regulates cell signaling. The term AMPylation was adopted in this work because addition of AMP could be an important PTM, it avoids confusion caused by adenylylation versus adenylation, and furthermore, it clearly differentiates the "addition of AMP" from other potential PTMs containing adenine/adenosine groups, for example, adenosine biphosphate (ADP) and adenosine triphosphate (ATP).

Received: 27 July 2010

Revised: 12 January 2011

Accepted: 13 January 2011

Published online: 24 February 2011 
Methods commonly used to map enzyme AMPylation sites include radioactive labeling [1, 10-13], gel electrophoresis analysis [1, 11], Edman degradation [10,11], and X-ray crystallography [13]. Only a handful of studies involve mass spectrometry [2, 4, 14-17], and most of these are only at the MS level. Thogersen et al. located the region of the AMPylation site by using fast-atom bombardment mass spectrometry to measure the mass of the chymotryptic peptides of the T4 RNA ligase, which had been AMPylated with ${ }^{14} \mathrm{C} \gamma$ ATP [2]. By comparing the matrix assisted laser desorption ionization-time of flight mass spectrometry (MALDI-TOF MS) spectrum of the unmodified GlnK protein with that of AMPylated GlnK protein, another group found a peptide with a mass increment of $329 \mathrm{Da}$ for modified GlnK [14], which is consistent with the shift expected following the addition of AMP. ESI-MS has also been used to monitor the AMPylation and de-AMPylation of bacterial glutamine synthetases [15]. Even fewer studies have been performed at the tandem mass spectrometry (MS/MS) level [4, 16, 17]. Leclercq and coworkers characterized the structure of AMPylated lincomycin [16] and clindamycin [17], but not the AMPylated peptide. Billy et al. reported that they obtained almost identical nanoESI-MS/MS spectra from AMPylated peptide and its non-AMPylated form [4]. The only difference was that two peaks were absent in the MS/MS spectrum of the latter: the precursor ion and its loss of $\left[\mathrm{AMP}-\mathrm{H}_{2} \mathrm{O}\right]$ form. None of these studies mapped the AMPylation site by mass spectrometry alone.

Although the structure of kemptide-AMP was not studied, Gibson et al. examined the tandem collision induced dissociation (CID) spectra of kemptide-ADP and kemptideATP, in addition to LSI-MS (liquid secondary ionization mass spectrometry) and MALDI-MS, to confirm that the AMPylated kemptides they synthesized had the desired structure [18]. They detected a series of fragment ions originating from the ADP and ATP groups, while the peptide sequence ions were insufficient to determine the position of the ADP/ATP modification site.

Previously, we analyzed wild-type (WT) Rac as well as Rac co-expressed with VopS (Rac/VopS) [5]. When the proteins were digested with AspN, we found that the mass of a peptide from Rac/VopS (aa 11-37, doubly charged peak at $m / z$ 1653.3) had a mass increment of 329.1 Da compared with that of the peptide from the WT Rac (aa 11-37, doubly charged peak at $m / z$ 1488.7). The MS/MS data of this modified peptide showed two forms of mass shift when compared with that of the WT peptide: $-18.0 \mathrm{Da}$ and +329.1 Da. From the position where the mass shift occurred, we believed that Thr35 of Rac was the modification site [5]. We then confirmed Thr35 was AMPylated by site mutation and radioactive labeling experiments [5]. Worby et al., in subsequent experiments, used tandem mass spectrometry data to identify the addition of AMP on a Tyr residue [8]. They reported the loss of adenine or adenosine and used that to determine the modification site.
Despite efforts mentioned above, systematic study has not previously been performed on the fragmentation pattern of peptides modified with AMP. Mass spectrometry based proteomics, as a powerful tool to identify PTMs, relies on the understanding of peptide fragmentation patterns, which could be used to develop refined bioinformatic algorithms for database searching [19]. Knowledge of its characteristic fragmentation pattern offers important benefits when mapping the AMP site on a protein by mass spectrometry. Although AMP as a potential PTM has only been found to occur on Thr [5] and Tyr [8,9], Ser AMPylation was included in this study since nucleophilic substitution of hydroxyl groups is a common mechanism for PTMs. In this work, we studied the fragmentation pattern of chemically synthesized model peptides containing AMPylated Thr, Ser, and Tyr, to better understand their characteristic dissociation products, and thus make it possible to confidently identify the AMPylation site by a high sensitivity mass spectrometry approach. We also studied the relative peak intensity of characteristic ions at varied collision energy (CE) levels, to offer helpful information on the design of experiments such as precursor ion scan, neutral loss scan and multiple reaction monitoring (MRM).

\section{Experimental}

\section{Peptides Studied}

The information on model peptides is summarized in Table 1. The AMPylated site was indicated by adding (amp) following the site of modification. To differentiate AMP group from the amino acid residues of the peptide, amp (lower case) was used.

\section{Peptide Synthesis}

Fmoc amino acids were used except for the N-terminal amino acid which was either Boc-Glu or Boc-Ala [20]. Side chain unprotected Fmoc Ser/Thr derivatives were used at the site of AMPylation for Ser(amp) and Thr(amp) sequences and Fmoc $\operatorname{Tyr}\left(\mathrm{PO}_{4}\right)$ was used in the case of $\operatorname{Tyr}(\mathrm{amp})$ peptides. The AMPylation reactions were performed as described previously [21].

\section{MS and MS/MS Analysis of AMP Standard and Model Peptides}

A dilution solvent with acetonitrile:water:formic acid (50:50:0.1 vol $/ \mathrm{vol})$ was used to make all solutions used in this study. A reference sample of AMP (Sigma-Aldrich, St. Louis, MO, USA) was dissolved in the dilution solvent to form a $10 \mathrm{ng} / \mu \mathrm{l}$ solution. The concentration of each model peptide solution was $10 \mathrm{ng} / \mu \mathrm{L}$.

The sample solution was directly infused using Proxeon Biosystems nanospray emitters (Odense, Denmark) and spectra were collected on a SCIEX QSTAR XL Q-TOF 
Table 1. Summary of information on model peptides

\begin{tabular}{lccc}
\hline \multicolumn{1}{c}{ Sequence } & Monoisotopic mass $(\mathrm{Da})$ & Singly charged ion detected $(\mathrm{m} / \mathrm{z})$ & Doubly charged ion detected $(\mathrm{m} / \mathrm{z})$ \\
\hline AAAHAAATVHV & 1017.53 & 1018.54 & 509.77 \\
AAAHAAAT(amp)VHV & 1346.58 & 1347.58 & 674.30 \\
AAAHAAASVHV & 1003.52 & 1004.52 & 502.76 \\
AAAHAAAS(amp)VHV & 1332.57 & 1333.58 & 667.30 \\
AAAHAAAYVHV & 1079.55 & 1080.56 & 540.78 \\
AAAHAAAY(amp)VHV & 1408.60 & 1409.60 & 705.30 \\
EYIPTVF & 867.44 & 1197.50 & - \\
EYIPT(amp)VF & 1196.49 & 1197.50 & 599.26 \\
EY(amp)IPTVF & 1196.49 & & 599.26 \\
\hline
\end{tabular}

mass spectrometer (Applied Biosystems, Framingham, MA, USA). For AMP standard reagent from Sigma, the mass range for both MS and MS/MS experiments was 50-1500 m/z. For model peptides, the MS mass range was $30-2000 \mathrm{~m} / z$, and $50-2000 \mathrm{~m} / \mathrm{z}$ for MS/MS experiments. For the MS/MS experiments, a unit resolution window was used. The goal was to keep the MS/MS spectra as clean as possible, while still being able to show the charge state of fragment ions. The majority of precursor ions passing through into the collision cell were ions with monoisotopic mass (M), and a portion with the mass of $(M+1)$. Precursor ions with the mass of $(M+2)$ or higher were not retained. The sum of $M$ and $(M+1)$ peak intensity, i.e., $\left.I_{M}+I_{(M}+1\right)$, was used to represent the peak intensity of a given peak in the MS/MS spectrum. The MS/MS spectra of unmodified peptides and their AMPylated counterparts were acquired under the same conditions, except that the CE for AMPylated peptide was $2 \mathrm{eV}$ higher than that for the unmodified peptide. Both MS and MS/MS spectra were obtained with 30 multiple channel acquisition (MCA) scans, while the accumulation time for each scan was $1.0 \mathrm{~s}$.
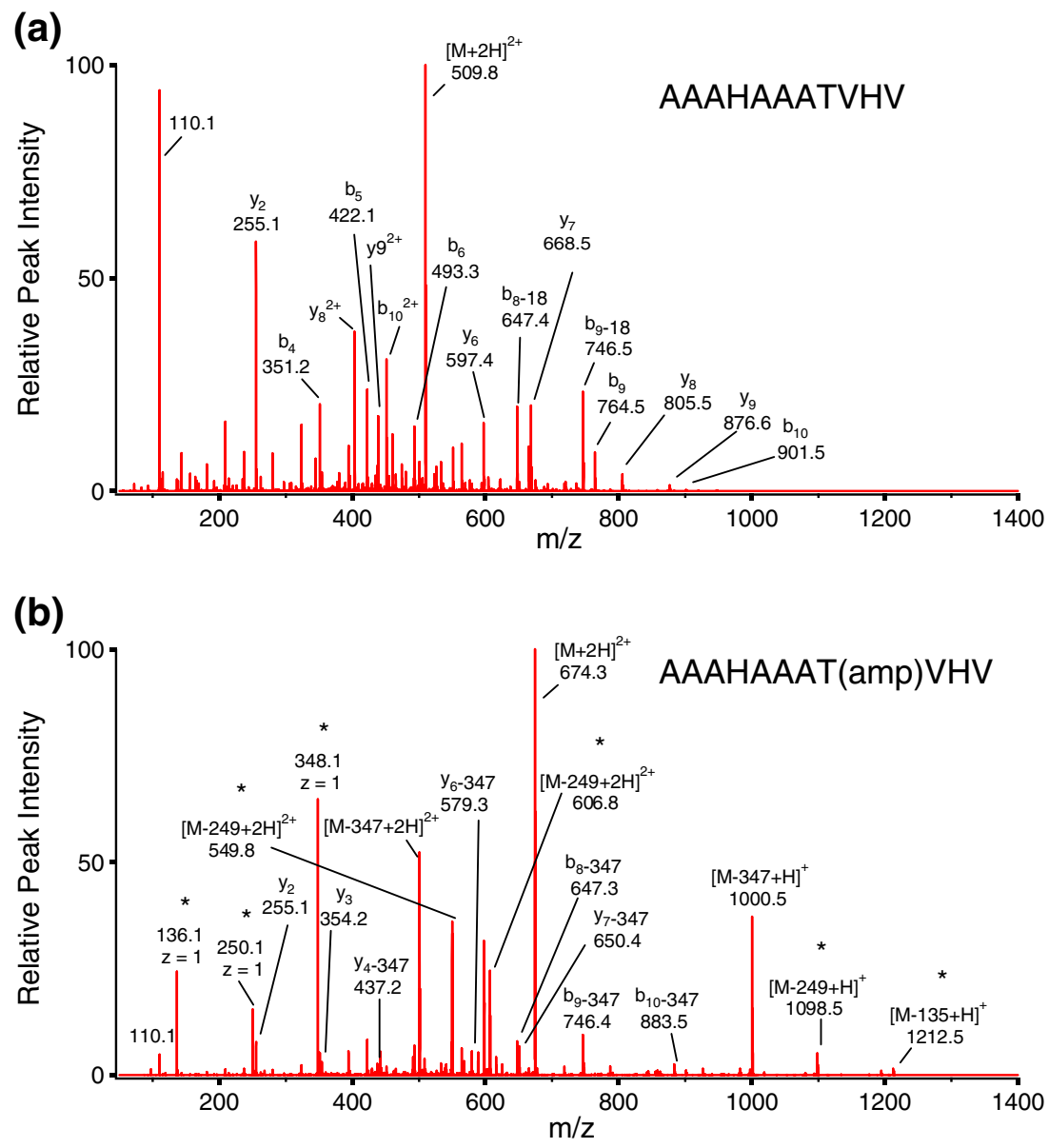

Figure 1. (continued) 

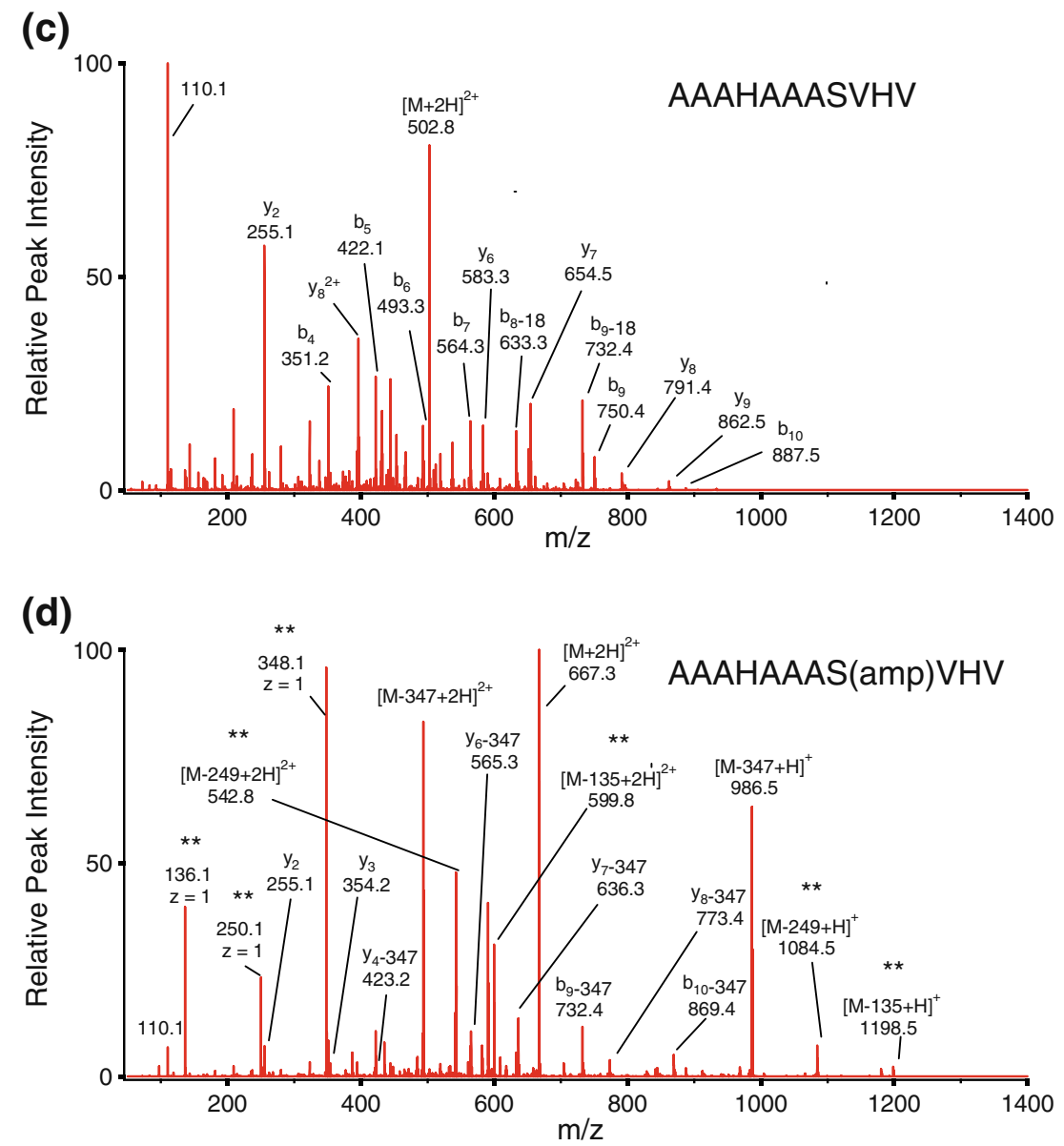

Figure 1. ESI-MS/MS spectra of model peptides. Although major ions were labeled, most doubly charged ions were not labeled to simplify the figure. (a) Unmodified peptide AAAHAAATVHV (CE=28 eV). (b) Model peptide with AMPylated Thr, AAAHAAAT(amp)VHV (CE=30 eV). Starting from the AMPylation site, y-ions showed a mass shift of -347 Da. Several other unique ions were detected only in the MS/MS spectrum of AAAHAAAT(amp)VHV. The major ones were marked with an asterisk *. (c) Unmodified AAAHAAASVHV (CE=28 eV). (d) Model peptide with AMPylated Ser, AAAHAAAS(amp)VHV (CE=30 eV). The mass shift of $-347 \mathrm{Da}$ occurred to fragments containing the AMPylated Ser residue. Other major unique ions detected were marked **

\section{Results and Discussion}

\section{Fragmentation Pattern of Model Peptides with AMPylated Thr/Ser}

In the MS/MS spectrum of peptide AAAHAAATVHV (Figure 1a), in addition to major $y$ - and b-fragments, several b-18 ions were detected due to the loss of $\mathrm{H}_{2} \mathrm{O}$, a commonly observed phenomenon for b-ions [19,22], while no y-18 ions were detected. For AAAHAAAT(amp)VHV (Figure 1b), once the fragments contained the AMPylation site, $y-347$ ions were detected with $\mathrm{S} / \mathrm{N}$ values varied between 10 and 200. The peak intensity of y-347 ions was generally higher than that of the corresponding y-ions. In addition to the $\mathrm{y}-347$ ions, several other unique ions were only detected in the MS/MS spectrum of peptide AAAHAAAT(amp)VHV (major ones were marked with an asterisk * in Figure 1b). Similarly, starting from the AMPylation site, y-347 ions and several other peaks were found

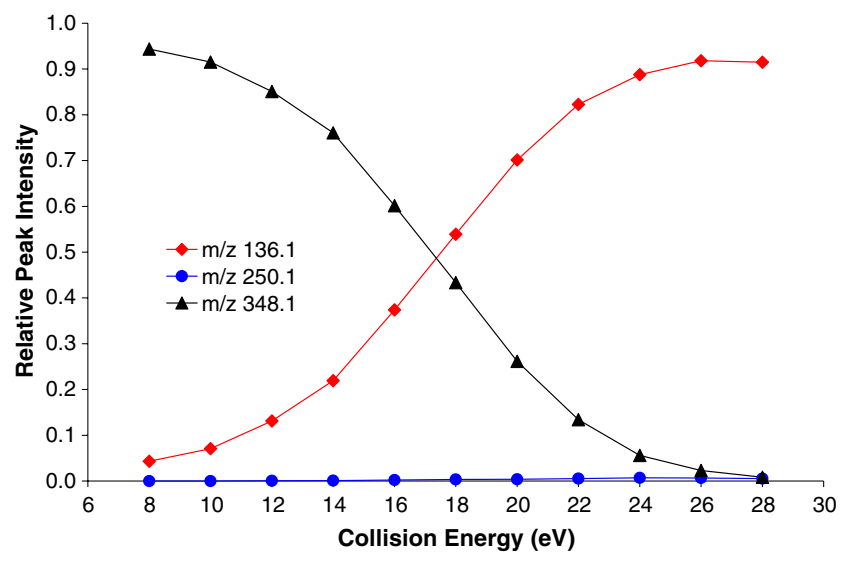

Figure 2. The relative peak intensity curve showed the fraction of each peak, i.e., $\Sigma$ (individual peak) $/ \Sigma$ (total peaks), as a function of CE 


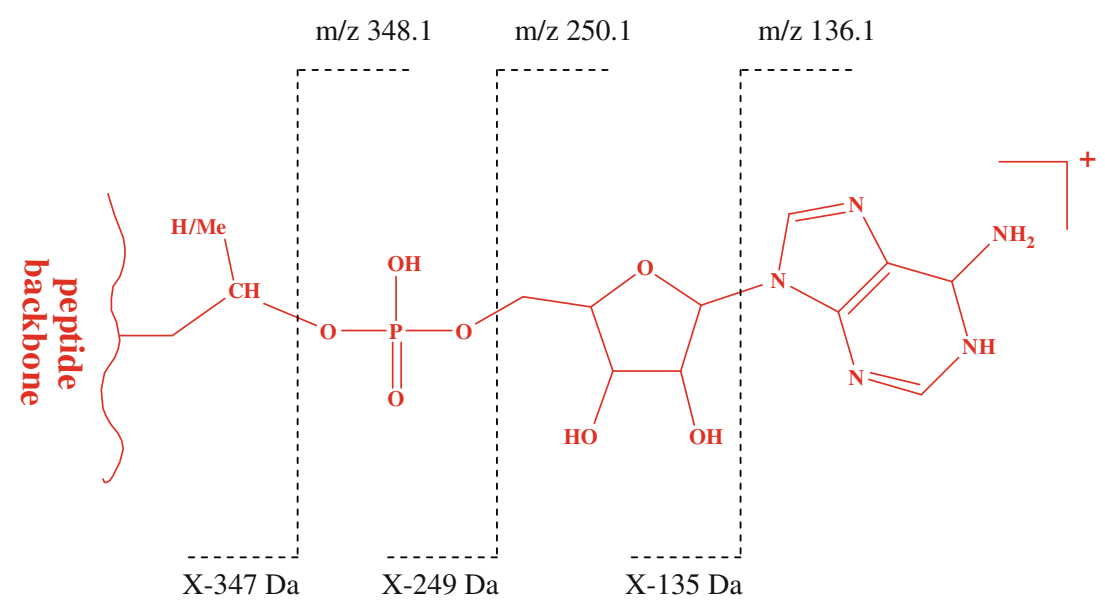

Scheme 1. Illustration showing the formation of unique ions detected in MS/MS spectra of AAAHAAAT(amp)VHV and AAAHAAAS(amp)VHV. "X" represents the mass of the modified peptide fragment

in AMPylated peptide (marked with ** in Figure 1d), but not in the unmodified peptide AAAHAAASVHV (Figure 1c). Unique peaks observed in both Figure $1 \mathrm{~b}$ and d were singly charged $\mathrm{m} / \mathrm{z}$ 136.1, 250.1, and 348.1. Given that AMP consists of a phosphate, a ribose, and an adenine moiety, and that masses of adenine and AMP are 135.1 and $347.1 \mathrm{Da}$, [adenine $+\mathrm{H}]^{+}$ and $[\mathrm{AMP}+\mathrm{H}]^{+}$could be the origin of peaks detected at $\mathrm{m} / \mathrm{z}$ 136.1 and 348.1, respectively. Detection of adenine represents the further fragmentation of the AMP group upon CID condition. Although fragmentation of the modification group itself was not commonly observed, the dissociation products of ADP and ATP were detected when kemptide-ADP and kemptide-ATP were subjected to CID experiments. In addition, the unique peak at $m / z 250$ shown in Figure $1 \mathrm{~b}$ and d was also found as one of the dissociation products of kemptide-ADP and kemptide-ATP [18].

To investigate the possibility of AMP fragmentation during the CID of AMPylated peptide, we performed the MS and MS/MS experiments on the pure reference AMP sample. AMP solution was infused into the mass spectrom- eter, and singly charged AMP, $[\text { AMP }+\mathrm{H}]^{1+}$, was detected at $\mathrm{m} / \mathrm{z}$ 348.1. No doubly charged peak was detected. Even when $\mathrm{CE}$ was set at $8 \mathrm{eV}$ to isolate the precursor ion, the product ion with $\mathrm{m} / \mathrm{z} 136.1$ was detected with $\mathrm{S} / \mathrm{N}>800$. Figure 2 showed the ratio of intensity of each peak to total intensity of all peaks in the MS/MS spectrum, i.e., $\Sigma$ (individual peak)/ $\Sigma$ total, as a function of CE. Gradually raising the $\mathrm{CE}$ from 8 to $28 \mathrm{eV}$, the $\Sigma(\mathrm{m} / z$ 136.1 $) / \Sigma$ total, increased from 0.04 to 0.92 . As the $\mathrm{CE}$ approached $12 \mathrm{eV}$, another product ion at $m / z 250.1$ became detectable $(\mathrm{S} / \mathrm{N}>$ 10). The AMP MS and MS/MS experiments showed that the AMP group could undergo dissociation upon CID MS/MS and generate fragments at $m / z 250.1$ and 136.1.

Scheme 1 illustrated the formation of unique ions detected in the MS/MS spectra of AAAHAAAT(amp)VHV and AAAHAAAS(amp)VHV. For simplicity, fragments carrying one charge were shown as an example. If the cleavage occurs at the $\mathrm{C}-\mathrm{O}$ phosphoester bond between the $\mathrm{Thr} / \mathrm{Ser}$ residue and the phosphate group, with the extra charge remaining on the peptide backbone, the fragments

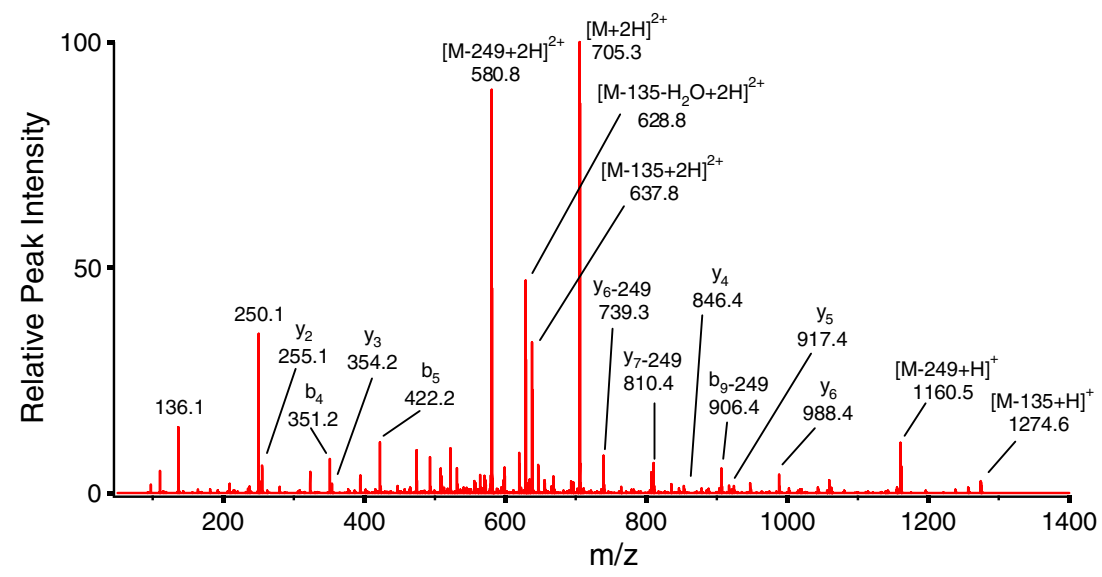

Figure 3. ESI-MS/MS spectra of model peptide AAAHAAAY(amp)VHV. Although major ions were labeled, most doubly charged ions were not labeled to simplify the figure. Peaks at $m / z 136.1$ and 250.1 , and several fragments with a mass shift of 135 or -249 Da were detected, while the peak at $m / z 348.1$ and $y-347$ ions were undetectable 
detected would have a mass shift of $-347 \mathrm{Da}$; with the charge on the AMP group, $[\mathrm{AMP}+\mathrm{H}]^{1+}$ would appear at $\mathrm{m} / \mathrm{z}$ 348.1. If the $\mathrm{C}-\mathrm{N}$ bond between ribose and adenine breaks, loss of adenine from the charged peptide fragments would result in a mass shift of $-135 \mathrm{Da}$, while [adenine $+\mathrm{H}]^{1+}$ would contribute to peak at $\mathrm{m} / z$ 136.1. So in Figure $1 \mathrm{~b}$, peaks at $m / z 1212.5(\mathrm{z}=1)$ and $m / z 606.8(\mathrm{z}=2)$ represented [AAAHAAAT(amp)VHV-adenine], and in Figure $1 \mathrm{~d}$, peaks at $m / z 1198.5(\mathrm{z}=1)$ and $m / z 599.8(\mathrm{z}=2)$ corresponded to [AAAHAAAS(amp)VHV-adenine]. If the cleavage occurred at the $\mathrm{C}-\mathrm{O}$ phosphoester bond between the phosphate group and the sugar, loss of adenosine would cause a mass shift of $-249 \mathrm{Da}$ for peptide fragments, while [adenosine$\left.\mathrm{H}_{2} \mathrm{O}+\mathrm{H}\right]^{1+}$ resulted in the peak at $m / z 250.1$. So, singly and doubly charged [AAAHAAAT(amp)VHV-adenosine] appeared at $m / z 1098.5$ and 549.8, respectively (Figure 1b), while [AAAHAAAS(amp)VHV-adenosine] ions accounted for peaks at $\mathrm{m} / \mathrm{z} 1084.5$ and 542.8 (Figure 1d). The proton affinity (PA) value of adenine $(942.8 \mathrm{~kJ} / \mathrm{mol})$ and adenosine $(989.3 \mathrm{~kJ} / \mathrm{mol})$ is higher than that of most amino acids ( $\sim 920 \mathrm{~kJ} / \mathrm{mol})$ except for His $(988 \mathrm{~kJ} / \mathrm{mol})$, Lys $(996 \mathrm{~kJ} / \mathrm{mol})$, and Arg (1051 kJ/mol) [23]. The PA value of adenine and adenosine may play a role in the readiness of formation of $[\mathrm{AMP}+\mathrm{H}]^{1+}$, [adenosine- $\left.\mathrm{H}_{2} \mathrm{O}+\mathrm{H}\right]^{1+}$, and [adenine $\left.+\mathrm{H}\right]^{1+}$.

During low energy CID MS/MS, fragmentations occur mostly along the backbone, resulting in predominantly b-, y-, and some a-series ions [24]. For peptides with AMPylated Ser/Thr, the large and labile AMP group is prone to leave the peptide and/or further fragment to lose adenine/adenosine, during CID MS/MS experiments. The combined effects of typical backbone cleavage with this special kind of side chain cleavage further complicated the MS/MS spectra of peptide with AMPylated Ser/Thr. The characteristic ions generated as a result are: fragments with AMP group attached; fragments with a mass shift of $-347,-249$, or $-135 \mathrm{Da}$; ions at $\mathrm{m} / \mathrm{z}$ 348.1, 250.1, and 136.1.

\section{Fragmentation Pattern of a Model Peptide with AMPylated Tyr}

In the MS/MS spectrum of model peptide AAAHAAAY (amp)VHV, while peaks at $m / z 136.1$ and 250.1 were still detected with $\mathrm{S} / \mathrm{N}$ ratio at least 50 , the peak at $\mathrm{m} / \mathrm{z} 348.1$ was no longer detectable $(\mathrm{S} / \mathrm{N}$ far less than 10 , generally $\mathrm{S} / \mathrm{N}<3$ ) (Figure 3). In addition, no y-347 ions were detected, and more fragments consistent with neutral loss of 135 or $249 \mathrm{Da}$ were found compared with MS/MS spectra of the model peptides with $\mathrm{Thr} / \mathrm{Ser}$ AMPylation.

Neutral loss of $\mathrm{H}_{3} \mathrm{PO}_{4}$ is dominant for phosphorylated Thr/ Ser, but not for fragments containing phosphorylated Tyr [25, 26]. Several pathways have been proposed for neutral loss of $\mathrm{H}_{3} \mathrm{PO}_{4}$ from Ser/Thr phosphorylation, including $\beta$-elimination $[25,26], \mathrm{S}_{\mathrm{N}} 2$-neighboring group participation and E2-elimination [27]. These pathways may also contribute to the loss of the AMP group from AMPylated Ser/Thr. However, all pathways mentioned above require the participation of the neighboring group, which is not possible with the phenolic ring of Tyr, which may explain the absence of the $y-347$ peak and the $m / z 348.1$ peak in the MS/MS spectrum of AAAHAAAY (amp)VHV. The neutral loss detected for phosphorylated Tyr
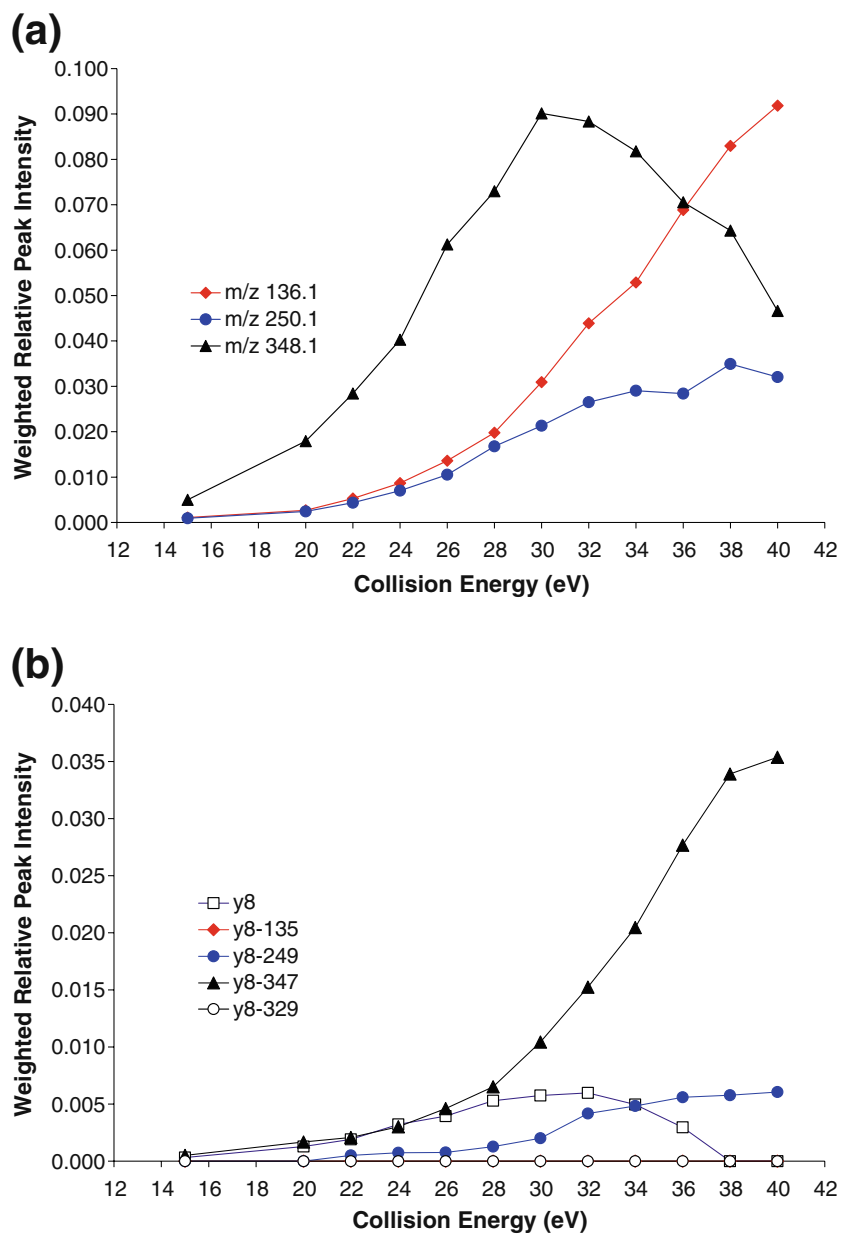

(c)

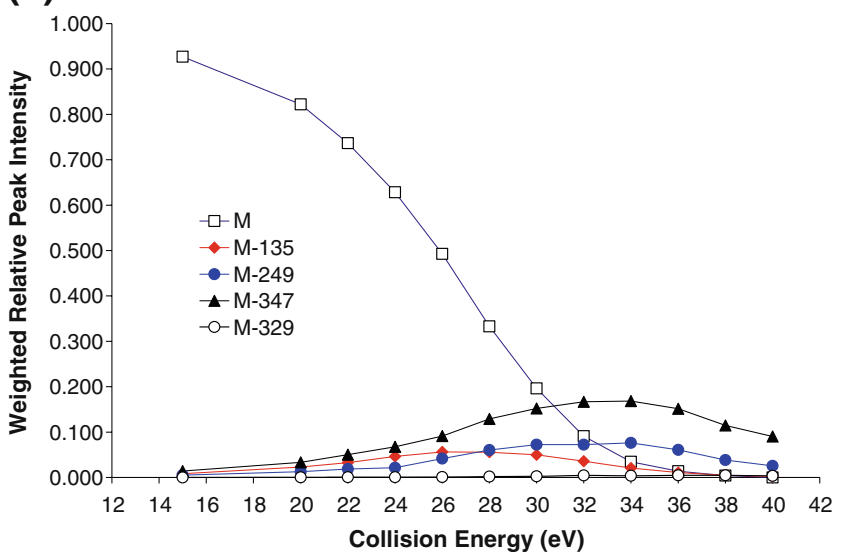

Figure 4. The intensity ratio of individual characteristic ions of AAAHAAAT(amp)VHV to total ions as a function of CE. (a) Characteristic ions with fixed mass: $\mathrm{m} / \mathrm{z}$ 136.1, 250.1, and 348.1. (b) Using $y_{8}$ related ions to represent $y$-ions. Peaks corresponding to $\mathrm{y}_{8}-135$ and $\mathrm{y}_{8}-329$ were undetectable at all CE applied. (c) Precursor related ions only. Peaks corresponding to [M-329] was undetectable at most CE applied 


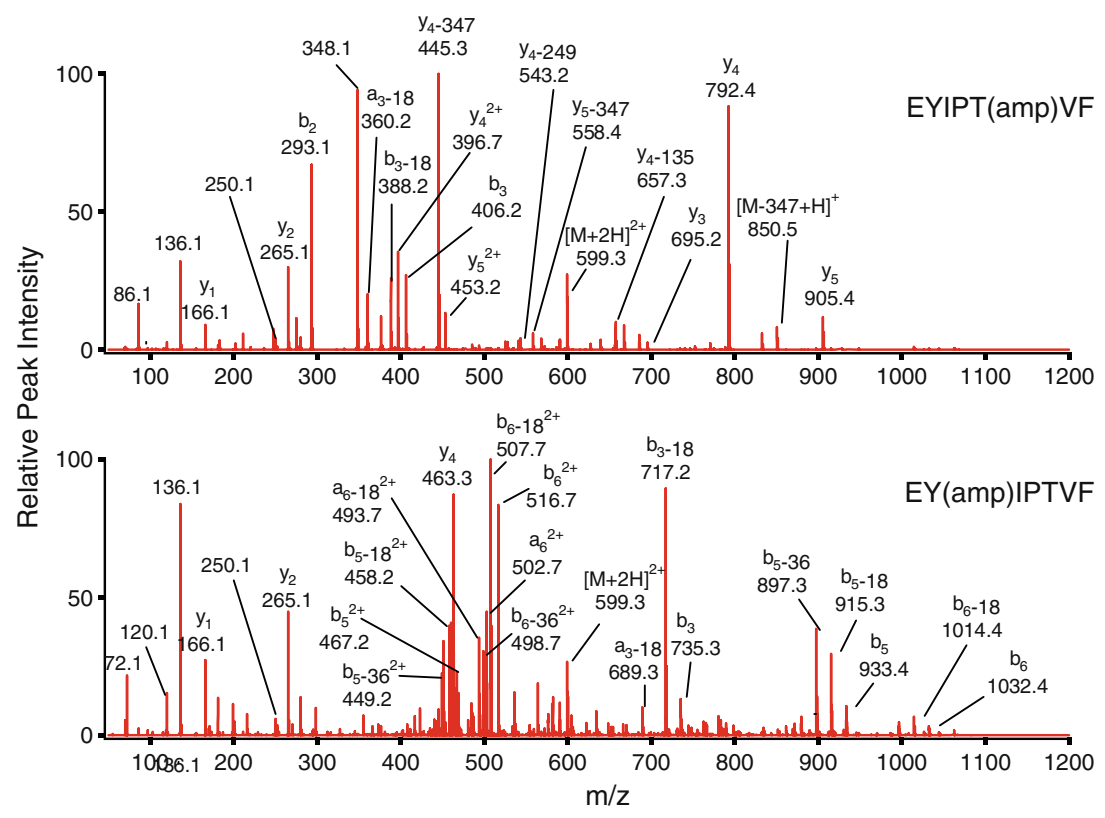

Figure 5. ESI-MS/MS spectra of model peptide EYIPT(amp)VF (top), and EY(amp)IPTVF (bottom)

was typically $\mathrm{HPO}_{3}$ [26]. However, no y-329 peaks were found in Figure 3. So for the peptide with AMPylated Tyr, the characteristic ions detected were: fragments with AMP group attached; fragments with a mass shift of -135 or $249 \mathrm{Da}$; peaks at $m / z 250.1$ and 136.1 .

\section{The Relative Peak Intensity of Characteristic Ions and the Influence of CE on the Detection of These Ions}

Characteristic ions at $\mathrm{m} / \mathrm{z} 136.1,250.1$, and 348.1 and the corresponding neutral loss of $135.1,249.1$, and $347.1 \mathrm{Da}$ are not sequence dependent, which theoretically makes them excellent candidates for precursor ion scan or neutral loss scan to selectively detect AMPylated peptides. In addition, these characteristic ions are of interest in MRM experiments. The insight gained by studying the relative peak intensity of characteristic ions and the impact CE has on their detection, will offer fundamental guides in the design of these experiments.

In order to compare the peak intensity of characteristic ions appearing in different MS/MS spectra, a weighted relative peak intensity was employed. In a MS/MS spectrum, the sum of intensity of all peaks (peaks with different charge states) belonging to a certain molecule (the precursor, or a fragment), $\Sigma$ (individual molecule), was used to represent the peak intensity of this molecule. Meanwhile, the sum of intensity of all peaks detected in this MS/MS spectrum was obtained, $\Sigma$ (all peaks). The ratio between $\Sigma$ (individual molecule) $/ \Sigma$ (all peaks) was the weighted relative peak intensity of this molecule. When a CE value was applied, $\Sigma$ (precursor) $/ \Sigma$ (all peaks) was used to indicate the portion of precursor ions that remain intact. Ideally, when a low CE is applied to isolate the precursor ions without causing any fragmentation, the $\Sigma$ (precursor) $/ \Sigma$ (all peaks) value is 1.0 , and the value of 0.0 means all precursor ions had fragmented.

AMPylated model peptides were fragmented with CE ranging from 15 to $42 \mathrm{eV}$, with the corresponding $\Sigma$ precursor/ $\Sigma$ (all peaks) ratio changing from $>0.90$ to $<0.02$. The results obtained for AAAHAAAT(amp)VHV and AAAHAAAS (amp)VHV were almost identical, so only the data obtained on AAAHAAAT(amp)VHV were shown (Figure 4). The weighted relative peak intensity of characteristic ions $(\mathrm{m} / \mathrm{z}$ 136.1, 250.1, and 348.1, and y-347, y-249, y-135, and y ions) were reported as a function of CE. Loss of $\mathrm{H}_{2} \mathrm{O}$ is common for b-ions (thus generating b-18 peaks), which would have complicated the analysis, so b-ions were not used in this study. The results calculated using the peak intensity of $y_{4}$ to $y_{10}$ were very similar to each other. We showed the data calculated with peak intensity of $\mathrm{y}_{8}$ related ions $\left(\mathrm{y}_{8}-347, \mathrm{y}_{8}-249, \mathrm{y}_{8}-135\right.$, and $\mathrm{y}_{8}$ in Figure $4 \mathrm{~b}$ ) because among all y-type fragments containing the AMPylation site, $y_{8}$ related fragments had the strongest peak intensity. The precursor related ions in Figure $4 \mathrm{c}$ were shown separately because peaks corresponded to [M-249] and [M-135] were much stronger than $\mathrm{y}_{8}-249$ and $\mathrm{y}_{8}-135$, respectively.

Figure 4a showed that for AAAHAAAT(amp)VHV, $\Sigma(\mathrm{m} / \mathrm{z}$ $348.1) / \Sigma$ (all peaks) reached the maximum value of $9.0 \%$ at $\mathrm{CE}=30 \mathrm{eV}$, then gradually decreased as $\mathrm{CE}$ increased. Raising CE always resulted in stronger peaks at $\mathrm{m} / z 136.1$ and 250.1. The order of the weighted average peak intensity was $\mathrm{m} / \mathrm{z} \quad 250.1<136.1<348.1$ when $\mathrm{CE}$ was smaller than $36 \mathrm{eV}$, where $\Sigma$ (precursor ions) $/ \Sigma$ (all peaks) was $1.4 \%$ (Figure 4c). However, the order was $\mathrm{m} / \mathrm{z}$ $250.1<348.1<136.1$ when $\mathrm{CE}>36 \mathrm{eV}$. Among y-type characteristic fragments, y-347 peak was dominant (Figure $4 \mathrm{~b}$ ). Even though $\mathrm{y}_{8}$ related ions were strongest among all y-type fragments, the peak intensity of $y_{8}-347$ 
was just comparable to that of $\mathrm{m} / \mathrm{z} 250.1$ for all CE applied (Figure $4 \mathrm{a}$ and $\mathrm{b}$ ). In Figure $4 \mathrm{~b}$, weighted intensity of $\mathrm{y}_{8}$ (the fragment with AMP group attached) reached the maximum at $\mathrm{CE} 32 \mathrm{eV}$, then rapidly decreased as $\mathrm{CE}$ was further increased, while the weighted peak intensity of y-347 and y-249 increased as CE was raised from 15 to $40 \mathrm{eV}$. Loss of AMP from precursor ions resulted in a strong [M-347] peak, stronger than $\mathrm{m} / \mathrm{z}$ 136.1 and 348.1. Although $\mathrm{y}_{8}-135$ was not detected and $\mathrm{y}_{8}-249$ was very weak, [M-135] and [M-249] peaks were detected with signal intensity greater than $\mathrm{y}_{8}-347$. Generally speaking, for all CE applied, $m / z$ 348.1, 136.1, and 250.1, fragments with AMP group attached, and fragments with a mass shift of -347 Da were major characteristic ions for peptide with AMPylated Ser/Thr residues. If the goal is to maximize signal strength for fragments with AMP group, optimizing CE is important. For model peptides, the $\mathrm{y}_{8}$ reached the maximum weighted peak intensity at the CE value where $\sim 90 \%$ of the precursor ion was fragmented. Characteristic fragments with a mass shift of -135 or -249 Da were comparatively weaker and were often undetectable, except for [M-135] and [M-249] ions of the model peptides.

Trends described for AAAHAAAT(amp)VHV held true for AAAHAAAY(amp)VHV, except that (1) neither y-347 peak nor $\mathrm{m} / \mathrm{z} 348.1$ was detected at any level of CE applied, (2) y-135 peak was clearly detectable in most cases, (3) with respect to AAAHAAAY(amp)VHV, CE needed was about $2 \mathrm{eV}$ higher to reach the same extent of fragmentation as AAAHAAAT(amp)VHV. The higher molecular weight of AAAHAAAY (amp)VHV may contribute to this need for a higher CE setting. Also, the loss of the AMP group is prohibited for Tyr AMPylation, and this may enhance the charge localization, given that the PA value of adenosine (a part of AMP) is larger than most amino acids except for His, Lys, and Arg.

\section{Validation of the Characteristic Ions and Their Relative Peak Intensity with EYIPT(amp)VF, and EY(amp)IPTVF}

It was previously demonstrated that Tyr32 and Thr35 of the Rac protein could be AMPylated [5, 8]. Model peptides EYIPT(amp)VF and EY(amp)IPTVF were used to validate the results obtained on AAAHAAAT(amp)VHV, AAAHAAAS(amp)VHV, and AAAHAAAY(amp)VHV. The sequence EYIPTVF corresponds to aa 31-37 region in the Rac protein. ESI-MS/MS spectra of EYIPT(amp)VF and EY (amp)IPTVF were shown in Figure 5. While peaks at $\mathrm{m} / \mathrm{z}$ 136.1, 250.1, and 348.1, together with $\mathrm{y}-347$ ions were detected in the MS/MS spectrum of EYIPT(amp)VF, the peak at $\mathrm{m} / \mathrm{z} 348.1$ and $\mathrm{y}-347$ ions were not found in that of EY(amp)IPTVF.

In Figure 6a, the weighted relative peak intensity of $\mathrm{m} / \mathrm{z}$ 136.1, 250.1, and 348.1 and peaks corresponded to [M-347], [M-249], [M-135], and precursor ions were reported as a
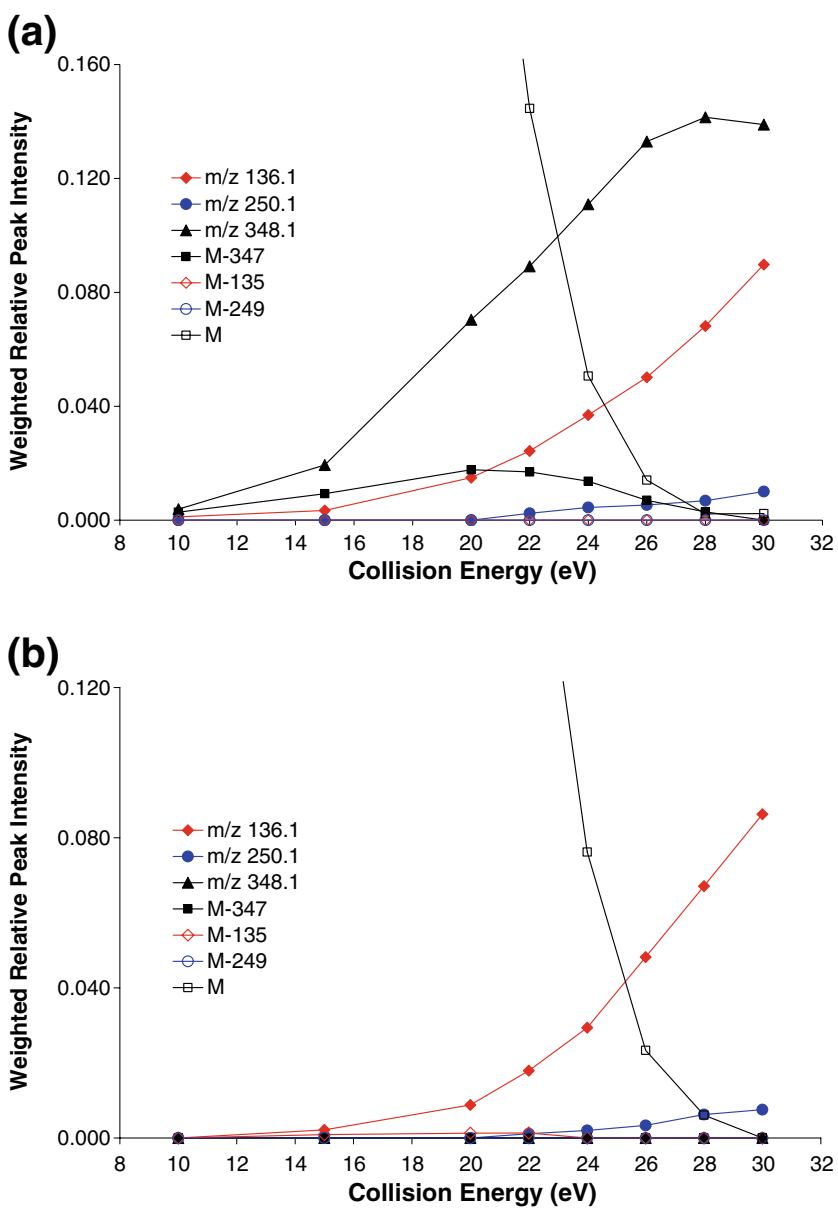

Figure 6. The intensity ratio of individual characteristic ions to total ions as a function of CE. (a) Peptide EYIPT(amp)VF. Although the intensity of peaks corresponding to [M-347] was comparable to that of $\mathrm{m} / \mathrm{z} 136.1$ when $\mathrm{CE}<22 \mathrm{eV}$, peaks corresponding to [M-249] and [M-135] were not detectable at all CE values applied. (b) Peptide EY(amp)IPTVF. Peaks corresponding to [M-135] were detected, but with much weaker signal compared with peak at $\mathrm{m} / \mathrm{z} 136.1$, and peaks corresponding to [M-347] and [M-249] were not detectable at all CE values applied

function of CE. Similar to the results described above, $\mathrm{m} / \mathrm{z}$ 348.1, 136.1, and 250.1, and fragments consistent with neutral loss of 347 Da were major characteristic ions, and raising CE always resulted in stronger peaks at $\mathrm{m} / z 136.1$ and 250.1. Similarly, y-135 and y-249 ions were weak and not always detectable (data not shown to simplify Figure 6). However, [M-249] and [M-135] peaks were strong when AAAHAAAT(amp)VHV and AAAHAAAS(amp)VHV were fragmented, but in the case of EYIPT(amp)VF, they were not detectable at all CE values applied. Again, for EY (amp)IPTVF (Figure 5 and Figure 6b), peaks at $\mathrm{m} / z 136.1$ and 250.1, and fragments with AMP group attached were major characteristic ions, while y-135 and y-249 ions, including [M-135] and [M-249], were much weaker and often undetectable. 
Characteristic ions at $\mathrm{m} / \mathrm{z} 136.1,250.1$, and 348.1 and the corresponding neutral loss of 135.1, 249.1, and 347.1 Da represent useful potential targets for selectively detecting AMPylated peptides. As for any method specifically targeting a PTM or a protein, there are obstacles that may hinder its application. First, other ions/molecules may have similar $\mathrm{m} / \mathrm{z}$ values or masses. For example, tyrosine immonium ion at $\mathrm{m} / \mathrm{z} 136.0762$ can be mistaken for the [adenine $+\mathrm{H}]^{1+}$ ion $(\mathrm{m} / \mathrm{z}$ 136.0623). The strategy to minimize the interference of non-AMPylated ions/molecules may include the use of combinations of characteristic ions/molecules described in this study. The same strategy is also useful to confidently identify the AMPylated peptides manually, given that the diversity of product ions of AMPylated peptides may present some combinatorial problems for database searching algorithms. Another potential obstacle is that for lower abundance precursors, the sheer diversity of products may reduce the sensitivity of MRM, or precursor ion scan experiments. The data shown in Figure 4 and Figure 6 provided some useful insights on how to address this problem. For example, $\mathrm{m} / \mathrm{z}$ 136.1, 250.1, and 348.1 (Figure 4a and Figure 6a) and y347 peaks (Figure 4b and Figure 6a) were dominant peaks, especially when CE was high enough to fragment $>90 \%$ of the precursor ion (Figure 4c). Monitoring those ions and using a $\mathrm{CE}$ value that fragments a higher percentage of the precursor ion may help to increase the sensitivity of MRM, neutral loss scan, or precursor ion scan experiments.

\section{Conclusion}

We performed a systematic study on fragmentation pattern of peptides containing AMPylated Thr, Ser, and Tyr. During CID MS/MS experiments, when the peptide was AMPylated on Ser/Thr residue, the AMP group was prone to leave the peptide. Meanwhile, AMP could also undergo further fragmentation to lose an adenine or an adenosine group. The PA values of adenine and adenosine are higher than those of most amino acids, which would facilitate the protonation of these leaving groups. The characteristic ions thus generated and detected were: $[\mathrm{AMP}+\mathrm{H}]^{1+}$ at $\mathrm{m} / z$ 348.1, $[\text { adenine }+\mathrm{H}]^{1+}$ at $m / z$ 136.1, and [adenosine- $\left.\mathrm{H}_{2} \mathrm{O}+\mathrm{H}\right]^{1+}$ at $\mathrm{m} / \mathrm{z}$ 250.1. Since these ions are independent of the peptide sequence, they represent promising targets for selectively detecting AMPylated peptides. Depending on the charge state of the peptide and the structure of its CID fragments, an extra proton(s) can be retained on peptide fragments and form other characteristic ions. The loss of an AMP, adenosine, or adenine group correspondingly generated fragments with a mass shift of $-347,-249$, or -135 Da, respectively.

The relative peak intensity of characteristic ions and the influence of CE were also investigated. Generally speaking, for peptides with AMPylated Ser/Thr, $m / z$ 348.1, 136.1, and 250.1, fragments with AMP group attached and fragments consistent with neutral loss of 347 Da were major characteristic ions, while diagnostic fragments consistent with neutral loss of $135 \mathrm{Da}$ or $250 \mathrm{Da}$ were weaker and not always detectable. Although for some peptides, [M-249] and [M135], peaks could be stronger than peaks corresponding to fragments with a mass shift of -249 or $-135 \mathrm{Da}$, respectively, this phenomenon does not apply to all peptides. For fragments with AMP attached, after the maximum intensity was reached, further $\mathrm{CE}$ increases diminished their signal.

The findings for Tyr AMPylation followed the same general patterns as those for Ser/Thr modification, with the exception that the neutral loss of AMP was not detected, so the characteristic ions of Tyr AMPylation did not include the peak at $\mathrm{m} / \mathrm{z} 348.1$, nor fragments with a mass shift of $347 \mathrm{Da}$. The characteristic ions demonstrated in this study can be used to confidently identify the AMPylation site based on MS and MS/MS data, and to selectively scan AMPylated peptides in complex protein mixtures.

\section{References}

1. Gumport, R.I., Lehman, I.R.: Structure of the DNA ligase-adenylate intermediate: lysine (epsilon-amino)-linked adenosine monophosphoramidate. Proc. Natl. Acad. Sci. USA 68, 2559-2563 (1971)

2. Thogersen, H.C., Morris, H.R., Rand, K.N., Gait, M.J.: Location of the adenylylation site in T4 RNA ligase. Eur. J. Biochem. 147, 325-329 (1985)

3. Belshaw, P.J., Walsh, C.T., Stachelhaus, T.: Aminoacyl-Coas as probes of condensation domain selectivity in nonribosomal peptide dynthesis. Science 284, 486-489 (1999)

4. Billy, E., Hess, D., Hofsteenge, J., Filipowicz, W.: Characterization of the Adenylation site in the RNA 3'-terminal phosphate cyclase from Escherichia coli. J. Biol. Chem. 274, 34955-34960 (1999)

5. Yarbrough, M.L., Li, Y., Kinch, L.N., Grishin, N.V., Ball, H.L., Orth, K.: AMPylation of Rho GTPases by Vibrio VopS disrupts effector binding and downstream signaling. Science 323, 269-272 (2009)

6. Yarbrough, M.L., Orth, K.: AMPylation is a new post-translational modification. Nat. Chem. Biol. 5, 378-379 (2009)

7. Haas, A.L., Warms, J.V., Rose, I.A.: Ubiquitin adenylate: structure and role in ubiquitin activation. Biochemistry 22, 438-4394 (1983)

8. Worby, C.A., Mattoo, S., Kruger, R.P., Corbeil, L.B., Koller, A., Mendez, J.C., Zekarias, B., Lazar, C., Dixon, J.E.: The Fic domain: regulation of cell signaling by adenylylation. Mol. Cell 34, 93-103 (2009)

9. Muller, M.P., Peters, H., Blümer, J., Blankenfeldt, W., Goody, R.S., Itzen, A.: The Legionella effector protein DrrA AMPylates the membrane traffic regulator Rab1b. Science 329, 946-949 (2010)

10. Heinrikson, R.L., Kingdon, H.S.: Primary structure of Escherichia coli glutamine synthetase. II. The complete amino acid sequence of a tryptic heneicosapeptide containing covalently bound adenylic acid. J. Biol. Chem. 246, 1099-1106 (1971)

11. Culp, J.S., Blytt, H.J., Hermodson, M., Butler, L.G.: Amino acid sequence of the active site peptide of bovine intestinal 5'-nucleotide phosphodiesterase and identification of the active site residue as threonine. J. Biol. Chem. 260, 8320-8324 (1985)

12. Stefan, C., Stalmans, W., Bollen, M.: Threonine autophosphorylation and nucleotidylation of the hepatic membrane protein PC-1. Eur. J. Biochem. 241, 338-342 (1996)

13. Lima, C.D., Klein, M.G., Hendrickson, W.A.: Structure-based analysis of catalysis and substrate definition in the HIT protein family. Science 278, 286-290 (1997)

14. Hesketh, A.R., Chandra, G., Shaw, A.D., Rowland, J.J., Kell, D.B., Bibb, M.J., Chater, K.F.: Primary and secondary metabolism, and posttranslational protein modifications, as portrayed by proteomic analysis of Streptomyces coelicolor. Mol. Microbiol. 46, 917-932 (2002)

15. Mehta, R., Pearson, J.T., Mahajan, S., Nath, A., Hickey, M.J., Sherman, D.R., Atkins, W.M.: Adenylylation and catalytic properties of Mycobacterium tuberculosis glutamine synthetase expressed in Escherichia coli versus mycobacteria. J. Biol. Chem. 279, 22477-22482 (2004)

16. Achard, A., Villers, C., Pichereau, V., Leclercq, R.: New $\operatorname{lnu}(\mathrm{C})$ gene conferring resistance to lincomycin by nucleotidylation in Streptococcus 
agalactiae UCN36. Antimicrob. Agents Chemother. 49, 2716-2719 (2005)

17. Petinaki, E., Guerin-Faublee, V., Pichereau, V., Villers, C., Achard, A., Malbruny, B., Leclercq, R.: Lincomycin resistance gene $\operatorname{lnu(D)}$ in Streptococcus uberis. Antimicrob. Agents Chemother. 52, 626-630 (2008)

18. Gibson, B.W., Medzihradszky, D., Hines, W.M., Auriola, S., Kenyon, G.L.: Mass-spectrometric characterization of a series of adenosylated peptides acting as bisubstrate analogs of protein-kinases. J. Am. Soc. Mass Spectrom. 5, 443-451 (1994)

19. Paizs, B., Suhai, S.: Fragmentation pathways of protonated peptides. Mass Spectrom. Rev. 24, 508-548 (2005)

20. Larsson, E., Luning, B.: Solid-phase phosphorylation of a peptide by the H-phosphonate method. Tetrahedron Lett. 35, 2737-2738 (1994)

21. Al-Eryani, R.A., Li, Y., Ball, H.L.: Chemical synthesis of Ser/Thr AM pylated peptides. Tetrahedron Lett. 51, 1730-1731 (2010)
22. Fu, Q., Li, L.: Neutral loss of water from the $b$ ions with histidine at the $\mathrm{C}$-Terminus and formation of the c ions involving lysine side chains. $J$. Mass Spectrom. 41, 1600-1607 (2006)

23. Hunter, E.P.L., Lias, S.G.: Evaluated gas phase basicities and proton affinities of molecules: an update. J. Phys. Chem. Ref. Data 27, 413-656 (1998)

24. Biemann, K.: Contributions of mass spectrometry to peptide and protein structure. Biomed. Environ. Mass Spectrom. 16, 99-111 (1988)

25. DeGnore, J.P., Qin, J.: Fragmentation of phosphopeptides in an ion trap mass spectrometer. J. Am. Soc. Mass Spectrom. 9, 1175-1188 (1998)

26. Tholey, A., Reed, J., Lehmann, W.D.: Electrospray tandem mass spectrometric studies of phosphopeptides and phosphopeptide analogues. J. Mass Spectrom. 34, 117-123 (1999)

27. Reid, G.E., Simpson, R.J., O'Hair, R.A.: Leaving group and gas phase neighboring group effects in the side chain losses from protonated derine and its derivatives. J. Am. Soc. Mass Spectrom. 11, 1047-1060 (2000) 